\title{
Security Provision in The Cloud Agent Architecture for Efficient User Authentication for Cloud Communication
}

\author{
S. Saritha \\ Department of CSE, M.Tech Student, JNTUCEA, Ananthapuramu, Andhra Pradesh, India
}

\begin{abstract}
Article Info
Volume 7, Issue 1

Page Number: 167-173

Publication Issue :

January-February-2021
\end{abstract}

\section{Article History}

Accepted : 01 Feb 2021

Published : 08 Feb 2021

\begin{abstract}
Agent-based Inter Cloud monetary model for reinforcing customer- to-cloud course of action and cloud to cloud trade was first raised, this work offers a response for this new and testing issue by detailing 1 ) novel shows that demonstrate the guidelines of relationship between purchaser subject matter experts and Cloud administrators, and among Cloud administrators, and 2) novel frameworks that oversee client administrators and Cloud experts in making the best decisions. First the customer needs to move their records into cloud. On that time cloud expert community as of now buy a space from cloud, by then the CSP(Cloud Service Provider) gives the customer name and secret key to buyer. Every Entity is independently checked by the verification. After the effective confirmation of client, the Cloud Agent set up a Secure Communication Protocol between the Client and Provider. The Data is shipped off the cloud specialist for check and approval. When the Cloud Agent, Authorizes the information it is shipped off the cloud for capacity. The Cloud Agent is likewise allotted with proficient checking of dynamic assets. Each customer can introduce its organization sales to various fogs and each cloud can recognize requests from various clients, Many-to-various game plan model for trading cloud resources. It contains purchaser specialists and cloud provider administrators following up to support customers and fogs independently. In a cloud market, PAs deal with CAs to develop organization level Agreement for satisfying organization necessities from consumers.

Keywords : Cloud computing, Cloud -to -cloud Interaction, Trade off, Concession making.
\end{abstract}

\section{INTRODUCTION}

Distributed computing is a kind of figuring that depends on sharing processing assets as opposed to having neighborhood workers or individual gadgets to deal with applications. Distributed computing is similar to lattice registering, a sort of figuring where unused preparing patterns of all PCs in an organization are saddles to be taken care of issues excessively serious for any independent machine.

From the viewpoint of multi-specialist frameworks, dynamic checking is favored over inactive observing since ACA(Autonomic Cloud Agents) are planned with a more significant level of self-rule, i.e., they can choose when to proactively send data about the

Copyright: (C) the author(s), publisher and licensee Technoscience Academy. This is an open-access article distributed under the terms of the Creative Commons Attribution Non-Commercial License, which permits unrestricted noncommercial use, distribution, and reproduction in any medium, provided the original work is properly cited 
conditions of their assets to ACM(Autonomic Cloud Managers). In any case, despite the fact that system is proposed for dynamic asset checking, they didn't devise a correspondence convention for ACAs and ACMs to share data. Then again, specialists in receive the CPRP to adaptively change the time spans for checking asset states dependent on various check reactions. Though CPRP can decrease the quantity of messages traded by adaptively changing the time spans for checking asset states dependent on various reactions, there might be tradeoff in the precision of the asset states because of sudden and unanticipated disappointments.

In distributed computing, the word cloud (also communicated as "the cloud") is used as a similarity for "the Internet," so the articulation disseminated registering connotes "a sort of Internet-based handling," where different organizations are passed on to an affiliation's PCs and contraptions through the web.

An operator based world view for building programming devices and test beds for cloud as set the board. Creating Cloud shows that specialist based arrangement components can be successfully embraced for supporting cloud administration exchange, cloud business and mechanizing Cloud administration synthesis. To help Cloud business, the work conceived an unpredictable Cloud arrangement component that supports equal exchange exercises in interrelated business sectors that is a Cloud administration market between shopper specialists and merchant operators, and different cloud asset markets between dealer operators and supplier operators. To mechanize Cloud administration creation, specialists in this work receive an engaged choice agreement net convention(FSCNP) for progressively choosing Cloud administrations and use administration ability tables(SCT) to record the run down of Cloud operators and their administrations.
The Intercloud is an interconnected worldwide "haze of mists" and an expansion of the Internet "organization of organizations" on which it is based. The term was first utilized with regards to distributed computing in 2007 when Kevin Kelly believed that "in the long run we'll have the intercloud, the haze of mists". It got well known in late 2008 and has additionally been utilized to portray the datacenter of things to come.

In man-made reasoning, a keen operator (IA) is a self-governing element which sees through sensors and follows up on a climate utilizing actuators (for example it is a specialist) and coordinates its movement towards accomplishing. Canny specialists may likewise learn or utilize information to accomplish their objectives. They might be extremely straight forward or exceptionally perplexing: a reflex machine, for example, an indoor regulator is an astute specialist.

An unfamiliar specialist is any individual who effectively does the interests of a far off nation while situated in another host nation, by and large external the securities offered to those working in their official limit with regards to a conciliatory mission. Unfamiliar operators might be residents of the host nation.

A multi-specialist framework (M.A.S.) is an electronic framework made out of different connecting savvy operators inside a climate. Multioperator frameworks can be utilized to take care of issues that are troublesome or outlandish for an individual specialist or a solid framework to comprehend.

\section{RELATED STUDY}

Kwang Mong Sim [2] proposed an unpredictable exchange component that upholds arrangement exercises among three kinds of members in different 
interrelated business sectors. The unpredictable arrangement instrument comprises a BargainingPosition-Estimation (BPE) system for the multilateral exchanges among shopper and representative specialists in a help market and Regression-Based Coordination (RBC) technique for simultaneous dealings among merchant and supplier operators in various asset market. The arrangement results among representative and supplier specialists in an asset market can conceivably impact the exchange results among dealer and shopper operators in an assistance market.

Seokho Son and Kwang Mong Sim [4] proposed a philosophy for Cloud Service Reservations. In this clients and providers need to develop organization level game plans through trade. While it is fundamental for both a buyer and a provider to concur on the expense of an assistance and when to use the organization.

Kwang Mong Sim [2] proposed an unpredictable exchange component that upholds arrangement exercises among three kinds of members in different interrelated business sectors. The unpredictable arrangement instrument comprises a BargainingPosition-Estimation (BPE) system for the multilateral exchanges among shopper and representative specialists in a help market and Regression-Based Coordination (RBC) technique for simultaneous dealings among merchant and supplier operators in various asset market. The arrangement results among representative and supplier specialists in an asset market can conceivably impact the exchange results among dealer and shopper operators in an assistance market.

Seokho Son and Kwang Mong Sim [4] proposed a philosophy for Cloud Service Reservations. In this clients and providers need to develop organization level game plans through trade. While it is fundamental for both a buyer and a provider to concur on the expense of an assistance and when to use the organization. It presents a multi-issue exchange component to encourage PTNs between
Cloud operators and compromise among cost and time allotment utilities. It is intended to simultaneously cause various proposition in an arrangement round that to create a similar totaled utility, contrasting just regarding singular cost and schedule opening utilities. Another oddity in this proposition is detailing a novel time allotment utility capacity that describes inclinations for various schedule openings.

Ali Akramizadeh, et al [5] utilize the notable Qlearning in broad structure games which operators have a fixed need in real life choice. They additionally present another idea called cooperative $\mathrm{Q}$-values which not exclusively can be utilized in real life determination, prompting a subgame wonderful balance, yet in addition can be utilized in update rule which is end up being merged. Affiliated Q-values are the normal utility of a specialist in a game circumstance which is a gauge of the estimation of the subgame amazing harmony point.

David Bernstein, et al [7] have extended contributions to incorporate register related capacities, for example, virtual machines, stockpiling, and complete working framework administrations. The distributed computing configuration yields discoveries in geological appropriation, asset use proficiency, and foundation robotization. " of their own. Public and private mists offer their end These "public mists" have been imitated by IT sellers for organizations to construct "private mists shoppers a "pay more only as costs arise" model - an incredible move for registering, towards a utility model like the power framework, the phone framework, or all the more as of late the Internet.

David Bernstein, et al [7] have extended contributions to incorporate register related capacities, for example, virtual machines, stockpiling, and complete working framework administrations. The distributed computing configuration yields discoveries in geological appropriation, asset use proficiency, and foundation robotization. These "public mists" have been imitated by IT sellers for 
organizations to construct "private mists" of their own. Public and private mists offer their end shoppers a "pay more only as costs arise" model - an incredible move for registering, towards a utility model like the power framework, the phone framework, or all the more as of late the Internet.

Rajkumar Buyya, et al [8] presents a 21st century vision of processing; recognizes different registering standards promising to convey the vision of figuring utilities; characterizes Cloud figuring and gives the design to making market-situated Clouds by utilizing advances, for example, VMs; gives considerations on market-based asset the board methodologies that incorporate both client driven assistance the executives and computational danger the board to continue SLA arranged asset designation.

J. Octavio Gutierrez, et al [11] proposed an operator based way to deal with create benefits in multi-Cloud conditions for various kinds of Cloud administrations: once virtualized administrations, constant virtualized administrations, vertical administrations, and even administrations. Specialists are supplied with a semi recursive agreement net convention and administration ability tables to create administrations dependent on customer prerequisites. Observational results got from an administrator based proving ground show that experts in this work can: viably structure organizations to satisfy organization essentials, self-rulingly select organizations reliant on novel costs, enough adjust to constantly changing clients organization needs that trigger updates, and make organizations in various Clouds even with insufficient information about Cloud individuals.

Domenico Talia et al [12] discusses Cloud enrolling models and plans, their usage in equivalent and passed on applications, and examines analogies, differentiates and anticipated helpful energies between Cloud figuring and multi-administrator systems. This examination is lead having at the highest point of the need list the goal of realizing unrivaled complex systems and astute applications by using of Cloud structures and programming administrators. The intermixing of interests between multi-expert structures that need strong coursed establishments and Cloud figuring systems that need astute programming with dynamic, versatile, and independent direct can achieve new systems and applications.

\section{IMPLEMENTATION}

\section{A. ARCHITECTURE OF THE SYSTEM}

This framework is focused on Agent-based Cloud asset determination and arrangement conventions. Operators in connect will utilize the agreement net convention for Cloud asset choice and organization separately. created work operators and asset specialists for Cloud asset choice. Speaking to shoppers, endeavor to choose fitting assets by sending calls for recommendations for running positions.

Addressing a provider, as in fig1 every RA (Resource Agent) overviews a CFP(Call For Proposal) and a JA's(manager) necessities. Asset Agents that satisfy the basics answer to the JA with proposal for executing the work. The JA picks the RA with the best proposal (e.g., the most irrelevant cost) for executing the work and rejects each other recommendation. Specialist will screens and channels the data and sends it to the customer.

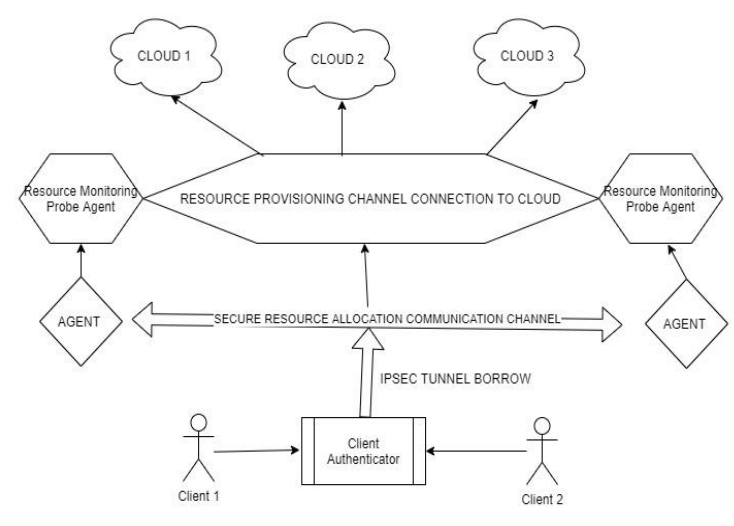

Fig 1: Architecture 


\section{B. Phase 1: Authentication:}

This module will keep the Data of User and Cloud Provider. Validation is a cycle where the qualifications gave are contrasted with those on document in a data set of approved clients' data on a neighborhood working framework. It may include affirming the character of an individual by approving their reports. In that User's and Cloud supplier subtleties like their name, email, contact number and area and so forth will be compared.

\section{Phase 2: File Storage:}

In this module, the customer moves the records with Respective provider and customer login information. Thus the customer archives can be seen by just explicit provider during the enlistment cycle. When the customer move their documents, can adjust open space.

D. Phase 3: Many to numerous Negotiation:

From the soonest point the customer can pick explicit Provider. The archives moved by the customers will be shipped off the specialist. If customer need more space, they can send space sales to the provider. In that module customer can change the provider. In that customer can pick the most diminished worth cloud space selling provider.

\section{E. Phase 4: Cloud Space Verification:}

In this module, checking the cloud space openness. In the occasion if the size of record moved by the customer is more unmistakable than the customer space open, at that point the bumble notice is shipped off the particular customer.

\section{F. Phase 5: Process of ABI(Agent Based Interface):}

In this, the cloud owner buys a space from abroad cloud owner, since, in such case that surmise $n$ number of provider needs to buy the cloud space. On that time cloud owner can offer space to the supplier.
The records transferred by the customers are reached to the specialist. When the specialist gets the records, at that point it confirms them. After confirmation the documents are shipped off the trustworthiness check, where the key is created for the real record and is coordinated with the key produced for the got record. In the event that both the keys are coordinated, at that point it is said that the record isn't tainted.

\section{G. Phase 6: Resource Allocation:}

In this, Provider designated the space to client and the cloud proprietor dispensed the space to the supplier. In the event that the client doesn't have the enough space can't allot the specific individual required space. On the time assign the breaking point space.

\section{H. Phase 7: Payment Gateway:}

In this module, client, supplier and cloud sending the space solicitation to specific authority as per their solicitation they will get the cloud space. When they finished at that point just added the cloud space to the specific client.

\section{Phase 8: Expired Notification:}

Customers once complete their portion by then added the space to the different customer accounts. By then they will start to move the records from the earliest starting point they will get cloud space with ended date. In this we will sent ended admonition before reach to slipped by date.

\section{J. METHOD}

Cloud - to - cloud Interaction Protocol and the consumer-to-Cloud exchange system embraces a novel multilateral (many-to-many) arrangement convention and a novel dealing procedure considered the ACR-MSC technique that is portrayed by both 1) versatile concession rate (ACR) and 2) negligibly adequate concession (MSC) . 
Method view examines about the data associated with document approval:

First the record is transferred by the customer to the cloud, however the document really doesn't arrive at the cloud without confirmation and approval at specialist level.

Specialist gets the document that is transferred by the customer and he select that specific record for confirmation, at that point the document is sent for the honesty check, where the document is scrambled and created a key. The key is cushioned with extra pieces. At that point the key is replicated for respectability check, on the off chance that the vital matches with the key created by the specialist, at that point the record is sheltered and it tends to be transferred to the cloud. On the off chance that the key doesn't coordinate with the specialists key, at that point the document it conclude that record was adulterated and it dismisses the document at specialist level.

Concession making calculation Consumers in a Cloud market vie for figuring associations and Cloud suppliers fight to offer kinds of help, a marketoriented approach considering the premium for and deftly of Cloud associations is fitting. Bargaining with cutoff times: Since customers overall have cutoff times in picking up enrolling resources for execute occupations and Clouds furthermore have cutoff times for booking their resources and executing occupations, the two Cloud Agents (CA) and Provider Agents (PA) are redone to make concessions with respect to time.

\section{EXPERIMENTAL RESULTS}

The Performance evaluation result is stated below In the under figure 2 Space Performance is talked about. In the chart $\mathrm{X}$-focus tends to time like a glimmer and Y-turn tends to Space Usage in KB. This diagram shows how much stockpiling will be utilized during the execution.

In the beneath figure 3: The entirety RAM is used by the framework during the report move is appeared in the diagram and it is spoken to in red line. The RAM utilized by the worker during the execution is appeared in the chart.

In the underneath figure 4: The CPU utilization is appeared. The percentage of CPU utilization during the execution is appeared. The chart is produced progressively dependent on the framework execution. Qualities in the fluctuates for various document move.

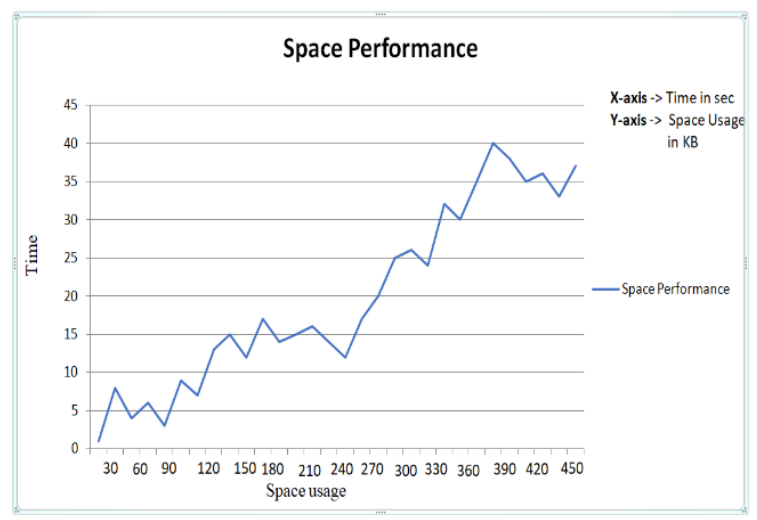

Fig 2: Space Performance Graph

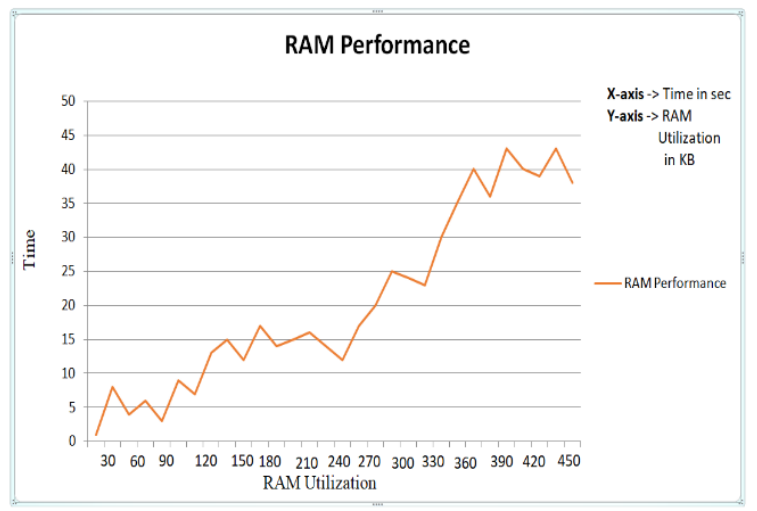

Fig 3: RAM Performance Graph 


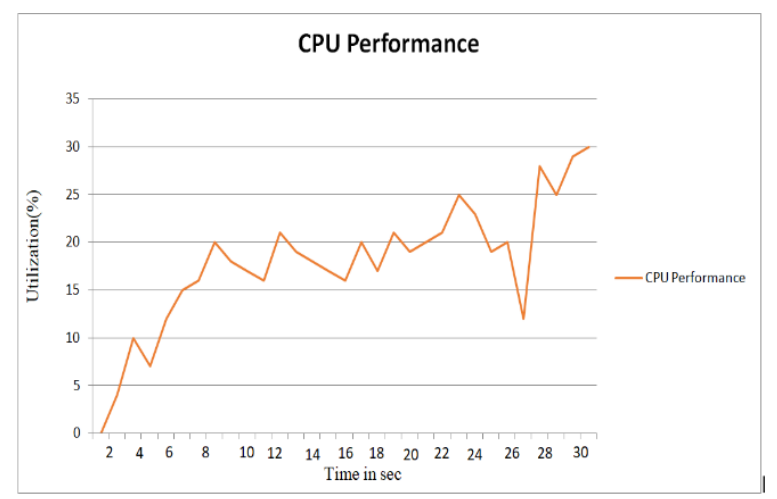

Fig 4: CPU Performance Graph

\section{CONCLUSION}

The primary use of this paper is to give secure correspondence among customer and cloud with the assistance of specialist, if the specialist doesn't approve any one customer he can be impeded. Principle utilization of this Paper is purchasing a cloud space from unfamiliar cloud proprietor. In this Paper we have added all the future work, as further future improvement the plan or building up of android application can be suggested because all the clients can't reach to the web administrations.

\section{REFERENCES}

[1]. K. M. Sim. Agent-based Cloud Commerce. Proc. IEEE Int. Conf. Industrial Eng. and Eng. Management 2009, Dec. 8-11, 2009, Hong Kong, pp. 717-721.

[2]. K. M. Sim. "Agent- based Cloud Computing," IEEE Transactions on Services Computing. vol. 5, no.4, Oct.- Dec., 2012, pp. 564- 577.

[3]. K. M. Sim, Complex and concurrent negotiations for multiple interrelated e- markets, IEEE Trans. Cybernet. 43 (1), pp. 230-245, 2013.

[4]. S. Son and K. M. Sim, A price- timeslot negotiation for cloud service reservation, IEEE Trans. Syst. Man Cybernet. B 42 (3), pp. 713728, 2012.

[5]. Ali Akramizadeh, Mohammad B. Menhaj and Ahmad Afshar Multiagent reinforcement learning in extensive form games with complete information.

[6]. D. Bernstein et al. Blueprint for the Intercloud Protocols and Formats for CloudComputing Interoperability. Proc. 4th Int. Conf. Internet and Web Applications and Services pp. 328-336., Venice, May 2009.

[7]. David Bernstein et al. An Intercloud Cloud Computing Economy - Technology, Governance, and Market Blueprints. Proc. 2011 Annual SRII Global Conference, pp.293- 299, San Jose, CA.

[8]. R. Buyya et al. Cloud computing and emerging IT platforms: Vision, hype, and reality for delivering computing as the 5th utility. Future Gener. Comput. Syst., 25(6): 599-616, Jun. 2009.

[9]. J.Ejarque et al. A Multi- agent approach for Semantic Resource Allocation. In IEEE Int. Conf. Cloud Computing Technology and Science, pp. 335-342, 2010.

[10].A. Dastjerdi and R. Buyya. An Autonomous Reliability- Aware Negotiation Strategy for Cloud Computing Environments. 2012 12th IEEE/ACM Int.Symp. on Cluster, Cloud and Grid Computing, May 13- 16, 2012, Ottawa, Canada, pp. 284- 291.

[11].J. O. Gutierrez- Garcia and K. M. Sim, Agentbased cloud service composition, Appl. Intell. 38 (3), pp. 436- 464, 2013.

[12].Domenico Talia ICAR-CNR \& University of Calabria Rende, Italy, Cloud Computing and Software Agents: Towards Cloud Intelligent Services.

\section{Cite this article as :}

S. Saritha, "Security Provision in The Cloud Agent Architecture for Efficient User Authentication for Cloud Communication", International Journal of Scientific Research in Computer Science, Engineering and Information Technology (IJSRCSEIT), ISSN : 2456-3307, Volume 7 Issue 1, pp. 167-173, JanuaryFebruary 2021. Available at doi : https://doi.org/10.32628/CSEIT217134 Journal URL : http://ijsrcseit.com/CSEIT217134 